\title{
Micro-CT as a well-established technique to investigate the internal damage state of a composite laminate subjected to fatigue
}

\author{
G. Chiesura*, G. Luyckx, E. Voet, W. Van Paepegem, J. Degrieck, \\ Department of Material Science and Engineering, Ghent University, Ghent, Belgium \\ M. N. Boone, J. Dhaene, L. Van Hoorebeke \\ UGCT - Department of Physics and Astronomy, Ghent University, Ghent, Belgium
}

\begin{abstract}
Micro Computed Tomography (micro-CT) has become an established non-destructive technique for microstructure investigation of composite materials, where due to the intrinsic difficulty in defining suitable damage models, it can be proved useful as such in helping to interpret and validate them. In this study the evolution of damage on several cross-ply laminates having embedded fibre optics of different diameters was investigated over several millions of fatigue cycles. High-resolution 3D X-ray tomography has been performed at different intervals in the vicinity of the sensor and damage has been quantified by counting the overall number of matrix cracks in the scanned volume. A clear evolution of damage has been noticed in accordance with a measured stiffness degradation of the laminate. Nevertheless, the results reveal that for the given loading conditions damage is not evolving in the vicinity of the fibre optics.
\end{abstract}

\section{INTRODUCTION}

When compared to metals, composite materials exhibit some interesting advantages as for instance, a higher stiffness-to-weight ratio, lower density and corrosion resistance. In addition, they exhibit a gradual stiffness degradation during in-life operation and not a sudden loss of properties as it happens for a metallic component when reaching its end-life. Furthermore, composite materials allow also the integration of sensors during their production. The latter can then be used during the whole lifetime of the component in order to gain information on its structural health. A desirable sensor technology which can be employed for this purpose is represented by fibre optics, which thanks to their small dimensions $(\approx 200 \mu \mathrm{m})$ and flexibility can be successfully embedded in between composite plies. This will allow for sensing the "real strain" to which the structure is subjected inside the composite laminate. However, questions arise when an optical fibre is embedded in a composite layup, e.g. what is the distortion created by the sensor in the surrounding composite layers? Will this distortion affect the structural integrity of the composite? Is the strain sensed by the fibre optic an artefact caused by the presence of the sensor itself? In this study, these questions are addressed via an experimental approach, where fibre optics were embedded in carbon fibres (CFRP) and glass fibres (GFRP) prepreg laminates and cycled for several millions of cycles under tensile loading. In order to investigate the internal state of the laminates, non- destructive assessments via micro-computed tomography (micro-CT or $\mu \mathrm{CT}$ ) were performed and damage in the scanned volume was traced and quantified. Some cracks were noticed at the fibre optic location directly after manufacturing in some carbon fibre samples: this was directly attributed to the relatively high $\left(180{ }^{\circ} \mathrm{C}\right)$ process temperature. Apart from this, no evidence of damage evolution on the fibre optics surroundings were found over several millions of fatigue cycles. High-resolution (i.e. 2 to $4 \mu \mathrm{m}$ voxel size) CT scans, both for carbon fibre and glass fibre composite, were obtained and the details around the fibre optic were highlighted. This technique has proven to be of relevance for internal material investigation and the damage assessment was proven by an overall stiffness decay measured on the sample. It has also to be noticed that the scans performed on the GFRP, presented more difficulties in defining the fibre optics outline. In fact, the difference in composition between the reinforcing fibres and optical fibre materials in this case was minimal (i.e. both optical fibre and reinforcing fibres have the same density and are made of glass), but this was achieved after an optimization of the scanning parameters.

\section{EXPERIMENTAL SET-UP}

\subsection{Sample production}

Two different laminates having both a $\left[90_{2}, 0_{2}\right]_{2 s}$ layup were produced by an autoclave process. The first 
one was a carbon-fibre UD prepreg M55J-M18 by Hexcel ${ }^{\circledR}$, which was cured for 2 hours at $180{ }^{\circ} \mathrm{C}$, imposing a vacuum level of $-80 \mathrm{kPa}$ and an external pressure of 7 bars. The second laminate was produced with MTM28 glass-fibre UD prepreg layers by Umeco® and was cured for 2 hours at $120{ }^{\circ} \mathrm{C}$, using vacuum level of $-80 \mathrm{kPa}$ and 5 bars of additional pressure. In the mid-plane of each laminate, optical fibres of different diameters (the outer diameter was ranging from $106 \mu \mathrm{m}$ to $195 \mu \mathrm{m}$ ) were aligned with the reinforcing fibres direction and embedded between two $0^{\circ}$ layers. For the GFRP laminate the fibre optics were carrying also a grating, which was positioned in the centre of the plate, while for the CFRP dummy fibres were used instead. After autoclave manufacturing, the laminates were cut to coupon size according to the ISO 527-4 standard. Due to the limitations imposed by the micro-CT setup (which will be explained in more detail in subparagraph 2.3) the carbon fibre samples had the following dimensions $t \times w \times l=2.5 \mathrm{~mm} \times 12 \mathrm{~mm} \times 250$ $m m$, while the glass fibre samples were $5 \mathrm{~mm} \times 19$ $m m \times 250 \mathrm{~mm}$. Aluminium tabs were glued to the specimens ends, allowing an overall gauge length of approx. $150 \mathrm{~mm}$.

\subsection{Fatigue loading set-up}

A preliminary set of static tests in accordance with the ASTM D3039 standard have been performed on three reference samples for each material in order to evaluate the Ultimate Tensile Strength (UTS) and therefore define the load amplitude of the fatigue cycles. The test procedure consisted of a set of cumulative cycle intervals, which were interspersed with static tensile tests; these tests served to evaluate the laminate stiffness and its reduction due to occurring damages. At this stage, the samples were also dismounted from the testing rig, moved to the $\mu \mathrm{CT}$ facility and scanned. Figure 1 summarizes the test procedure in a schematic.

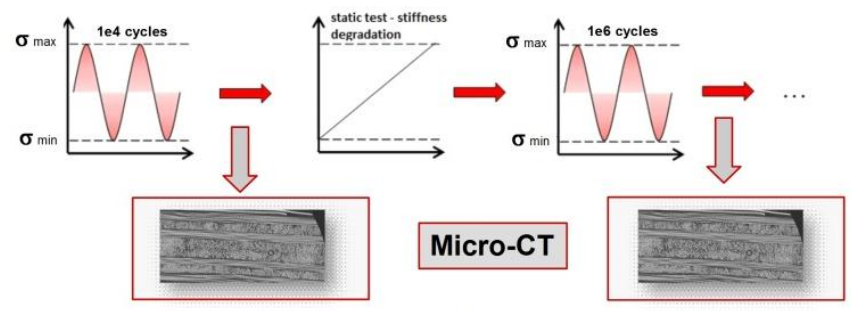

Testing Method

Figure 1. Schematic of the testing method: the fatigue test has been divided in several cycle intervals; between each of them a static test was performed and the sample was scanned for damage assessment.

The fatigue tests were performed in accordance with the ASTM D3479M standard. A tensiontension sinusoidal load controlled cycle was selected in order not to buckle the specimens, thus avoiding unwanted premature damage. As a testing rig, the $100 \mathrm{kN} 8801$ servo-hydraulic machine from Intron ${ }^{\circledR}$ was used. All the samples were tested at a cycle frequency of $5 \mathrm{~Hz}$, as a good compromise between testing time (which was of 55 hours for $10^{6}$ cycles) and the risk of overheating the samples with consequent matrix degradation. In fact, in a preliminary testing stage, the effect of the loading frequency was evaluated by measuring the temperature increase during fatigue cycling. Due to the relatively low strains levels and the cross-ply lay-up, no relevant increase of temperature was noticed. The CFRP samples were initially cycled between 50 and $450 \mathrm{MPa}(\sim 50 \%$ of the UTS) for a cumulative of 1,000 cycles. The cycle was then resumed at the same stress level till $1,000,000$ cycles. Afterwards the load level was increased to $600 \mathrm{MPa}$ ( $70 \%$ of the expected UTS) and cycled for another 1,000 cycles, resulting in a cumulative of 1,001,000 cycles. The following load cycles were continued at the same stress level till 2,000,000 cycles and, as last, till 4,000,000 cycles.

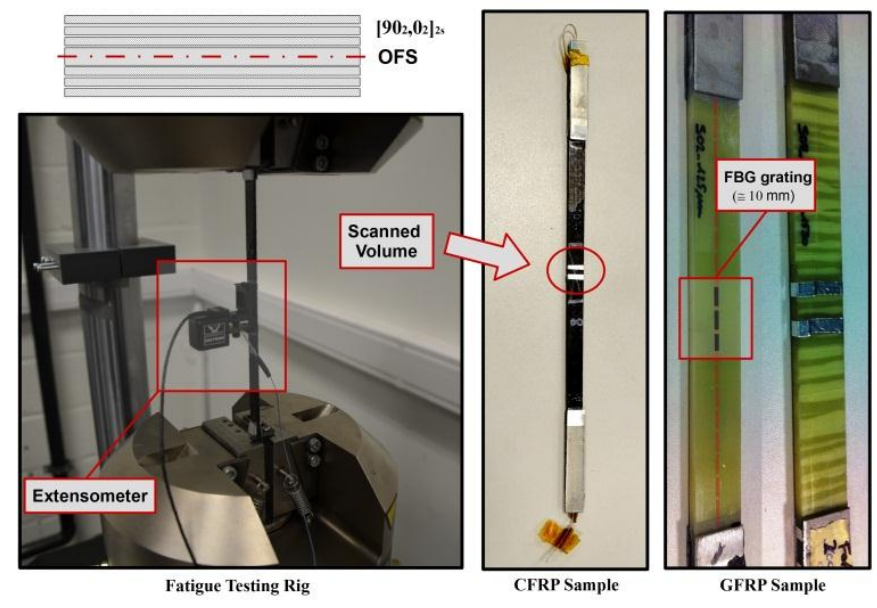

Figure 2. Testing setup: (top-left) schematic of the lay-up for the laminate and fibre optics embedding location, (bottom-left) sample mounted between the grips of the servo-hydraulic testing machine and (right) both carbon and glass fibres specimens prepared for testing.

For the GFRP samples instead, the amplitude was set between 30 and $130 \mathrm{MPa}(\sim 35 \%$ of the UTS) and kept for all the stages till 4,000,000 cycles. Tests were interrupted at 10,000, 1,000,000, 2,000,000 and $3,000,000$ cycles. The mean strain on the central region of the gauge length was recorded with a dynamic extensometer during the static tests. For the GFRP samples, the longitudinal strain on the outer $90^{\circ}$ layers was compared to the corresponding strain measured from the FBGs inside the laminate (between two $0^{\circ}$ layers). In Figure 2 the samples prepared for testing are depicted. One can notice the aluminium strips on the central gauge length region which were used as marks for the CT scans in order to scan repetitively the same volume. 


\subsection{CT-scanners}

As already mentioned in the previous subparagraph, the samples were scanned in the central region of their gauge length with a voxel size ranging from 2 to $4 \mu \mathrm{m}$. The obtainable 3D scan resolution is normally much lower than the voxel size, but with optimized scanning parameters, the latter can lead to a reasonable esteem of it (although there is no one to one correspondence). For the sake of simplicity, in the following the term resolution will be used. The scanned volume was of approx. $t \times w \times l=2 \times 4 \times 4$ $\mathrm{mm}^{3}$ for the $2 \mu \mathrm{m}$ voxel size scans and of $4 \times 8 \times 8$ $\mathrm{mm}^{3}$ for the $4 \mu \mathrm{m}$ ones, respectively. The scans were obtained with two different setups, property of the UGCT - Centre for X-ray Tomography of Ghent University. Both of them had an X-ray tube emitting $\mathrm{X}$-rays through the sample, which was fixed vertically on a rotational stage, and a flat panel detector to record digital radiographs. In Figure 3 the HECTOR (High-Energy CT scanner Optimized for Research) scanner is depicted. The X-ray tube is a high- energy XWT 240-SE microfocus source (from X-RAY WorX®) which was set at a Voltage of 140 $\mathrm{kV}$ and $8 \mathrm{~W}$ of target power, while maintaining a minimum focal spot size of approx. $4 \mu \mathrm{m}$. A 40x40 $\mathrm{cm}^{2}$ PerkinElmer $1620 \mathrm{CN} 3 \mathrm{CS}$ flat panel detector and a total of 9 motorized axes of freedom allows for a high flexibility scanning. Nevertheless, the high-precision stage mounted on the manipulator allows for fine positioning of the sample and therefore a spatial resolution up to $4 \mu \mathrm{m}$ is also achievable.

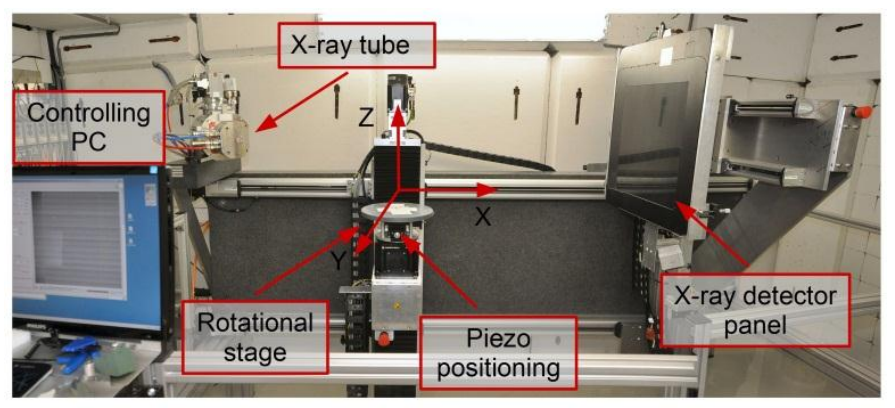

Figure 3. High-energy CT scanner optimized for research (Hector) used to scan at high resolution the composite specimens.

The second setup used is similar to the scanner just described but scaled-down in order to achieve better resolution up to $2 \mu \mathrm{m}$ on a smaller sample size. The X-ray tube, a medium energy FXE-160.50 dual head open type source (from Feinfocus ${ }^{\circledR}$ ). The detector used for these scans is a Varian $2520 \mathrm{~V}$ Paxscan a-Si flat panel with $1820 \times 1460$ pixels, 127 $\mu \mathrm{m}$ pixel size, covering a $20 \times 25 \mathrm{~cm}^{2}$ area. The sample manipulator is an XYZ-theta CT system (UPR-160F AIR) with ultra-precision air-bearing rotation motor from $\mathrm{PI} \mid \mathrm{miCos} \mathrm{GmbH}$.

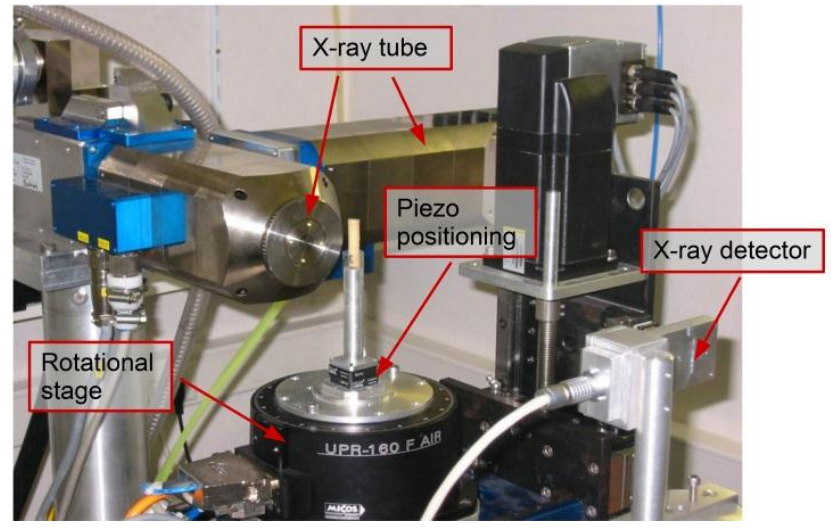

Figure 4. Modular $900 \mathrm{~nm}$ medium-energy micro-CT scanner used for the non-destructive evaluation of the composite samples.

The X-ray source was set at $100 \mathrm{kV}$ and $3 \mathrm{~W}$ of target power, resulting in a focal spot size of $2 \mu \mathrm{m}$. The source to object distance (SOD) was set to 14.4 $\mathrm{mm}(27.5 \mathrm{~mm}$ for the $4 \mu \mathrm{m}$ voxel size) and the source to detector distance (SDD) to approximately $870 \mathrm{~mm}$, resulting in a magnification around 60x and a corresponding voxel size of $2 \mu \mathrm{m}$. The stated distances, along with the conical X-ray beam, results in a geometric magnification, and the small focal spot size minimizes image smoothing. The reduction of spatial resolution ensures high image quality at this magnification. Moreover, the bigger the panel detector area is, the larger the obtainable magnification will be. On the other hand, one should also account for noise effects which could derive from the large SDD and a low energy of the outgoing beam (i.e. sample material with high attenuation). In some scans also a metal plate filter (e.g. aluminum or copper) was placed in between the source and the sample, with the aim of attenuating the higher energy beam component responsible for the so called beamhardening artifact. In a tomographic dataset, X-ray radiographs are acquired at different viewing angles. The stability of the scanning geometry, either due to sample movement or focal spot drift, is monitored during the CT scan by returning to a reference point a number of times. For the scans discussed in this paper, no shift was observed. In total 1500 projections were recorded over $360^{\circ}$ and the data were reconstructed with the in-house developed software package Octopus Reconstruction. The reconstructed pictures were post-processed through Matlab® and analyzed. The stack of cross-sections can be further employed to render the whole scanned volume. For this purpose, 3D renderings were made with VGStudio Max from the original reconstructed images.

\section{SCAN RESULTS}

\subsection{CT's cross-sections}

In this paragraph the cross-section images will be firstly introduced. Then, in the following section, the 
$3 \mathrm{D}$ renderings relative to the same scans will be presented. As already mentioned, these cross-sectional images were obtained from post-processing of a stack of 20 adjacent reconstructed slices; this allowed reducing the noise level and enhancing the contrast-to-noise-ratio, with consequent details smoothing. In Figure 5, the post-processed crosssection of a CFRP laminate having a $125 \mu \mathrm{m}$ embedded optical fibre $(195 \mu \mathrm{m}$ diameter considering its coating) is given. Clearly recognizable in the image are the differently oriented $0^{\circ}$ and $90^{\circ}$ plies, which are defining the cross-ply lay-up. Each $0^{\circ}$ or $90^{\circ}$ layer is composed by two prepreg plies; in the centre of the laminate, the fibre was placed in the middle of a four plies layer oriented at $0^{\circ}$. Most relevant to be noted, is the crack which has developed at the sensor/composite interface.

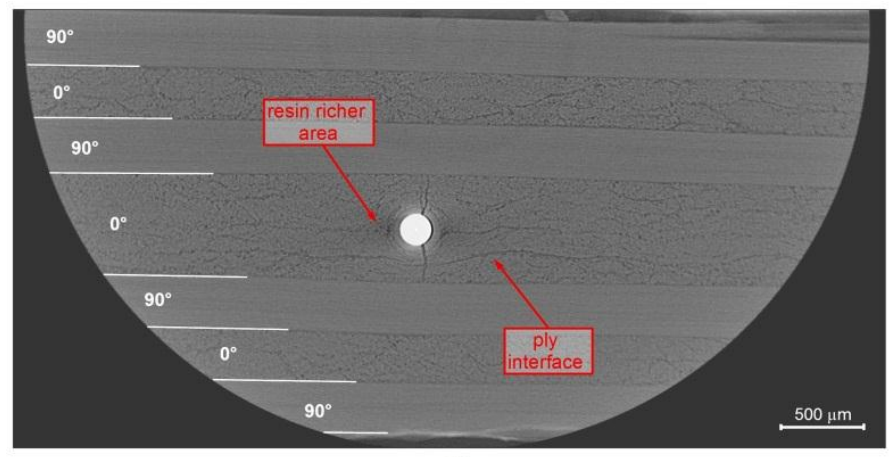

a)

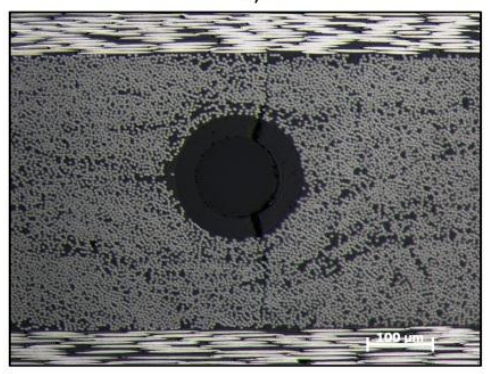

b)

Figure 5. (a) CT cross-section of a carbon fibre sample having a $125 \mu \mathrm{m}$ optical fibre embedded. (b) The scan is compared with the corresponding picture taken with a microscope after cutting and polishing the sample.

Comparing the fibre close-up with an optical microscopy image taken from a polished cross-section on a similar sample (Fig. 5b), one can notice some analogies. Firstly, the crack presents the exact same geometry in both images. The samples were analysed shortly after manufacturing and were not yet subjected to any mechanical loading. In other words, probably the stress build-up during curing of the prepreg layers has been sufficiently high to induce the damage. This is explainable by the low resistance of the composite plies in the transverse direction (which is governed only by the resin) and by the thermal expansion mismatch between the sensor and the hosting composite. Secondly, even the richer resin content interfaces between two adjacent plies can be distinguished on the fibre neighbourhood.
Particularly, it is noticeable how the plies are forced to redistribute around the sensor, resulting in resin richer areas sideways the fibre.

In Figure 6 the same post-processed image has been obtained for a GFRP sample having a $125 \mu \mathrm{m}$ fibre embedded. Two important findings are worth to be reported: the contrast and the quality of the scan have been proved high enough even though the composition of the reinforcing fibre (E-glass) and of the optical fibre is very similar. Moreover, no crack appeared on the sensor surroundings and this can be explained by the lower curing temperature imposed during manufacturing of the laminate. In addition to this, also the thickness of each prepreg layer for the MTM28 was approx. double the amount of the M55J-M18 (0.312 $\mathrm{mm}$ and $0.156 \mathrm{~mm}$, respectively), allowing the stresses surrounding the fibre to decrease. However, a similar scan was also done on a $(90,0)_{\mathrm{s}}$ cross-ply MTM28 prepreg sample (halved number of plies and total thickness of $2.5 \mathrm{~mm}$ ) and on the surroundings of the fibre $(195 \mu \mathrm{m}$ outer coating diameter) no cracks were found.

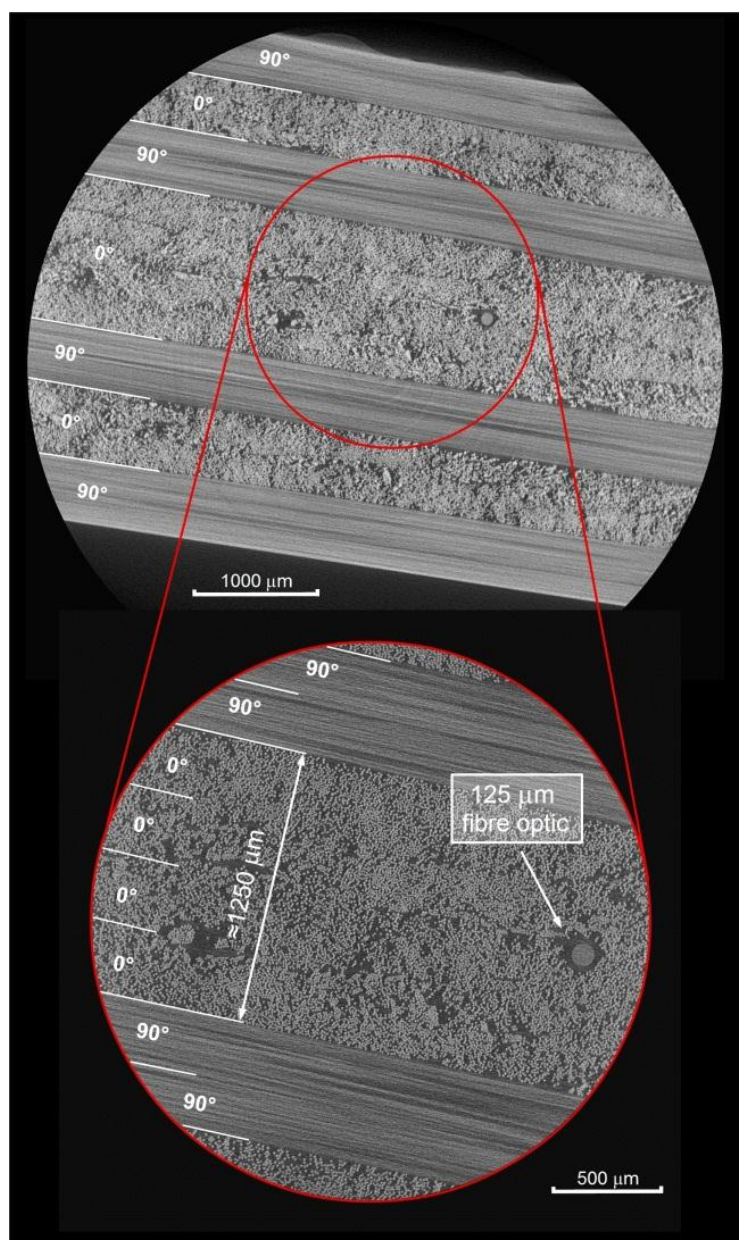

Figure 6. CT cross-section of a glass fibre sample having a 125 $\mu \mathrm{m}$ optical fibre embedded that was scanned at $4 \mu \mathrm{m}$ voxel size. The enlargement is the result of a finer scan obtained at 2 $\mu \mathrm{m}$ voxel size.

\section{$3.23 D$ renderings}

The reconstructed stack of cross-sections was rendered with VGStudio Max in a 3D volume of ap- 
prox. $t \times w \times l=2.5 \times 5 \times 5 \mathrm{~mm}^{3}$ for a $2 \mu \mathrm{m}$ voxel size scan (or $5 \times 5 \times 10 \mathrm{~mm}^{3}$ for a $4 \mu \mathrm{m}$ voxel size). This allowed to freely examining the surroundings of the fibre optic sensor and the adjacent composite layers in order to check for damage. As suspected the crack at the sensor/composite interface for the CFRP sample was running along the whole fibre, suggesting that it was not a casual and localized damage, but more a systematic effect instead, mostly related to a wrong combination of materials/manufacturing parameters as already mentioned in the previous subsection. In Figure 7 all this information is presented for a 3D rendering of a scan on a CFRP sample which, in this case, was loaded in fatigue for 2,000,000 cycles. In red colour the fibre optic, as well as the aluminium strips used to mark the scanning volume, are highlighted for clarity in the images. Besides the already mentioned crack evolving through the coating of the fibre optic, no additional damage was assessed on the sensor surroundings. Instead, damage was found on the $90^{\circ}$ plies as it is noticed on the top right side of the figure (Fig. 7b), where the transversal cracks have been highlighted in white boxes.

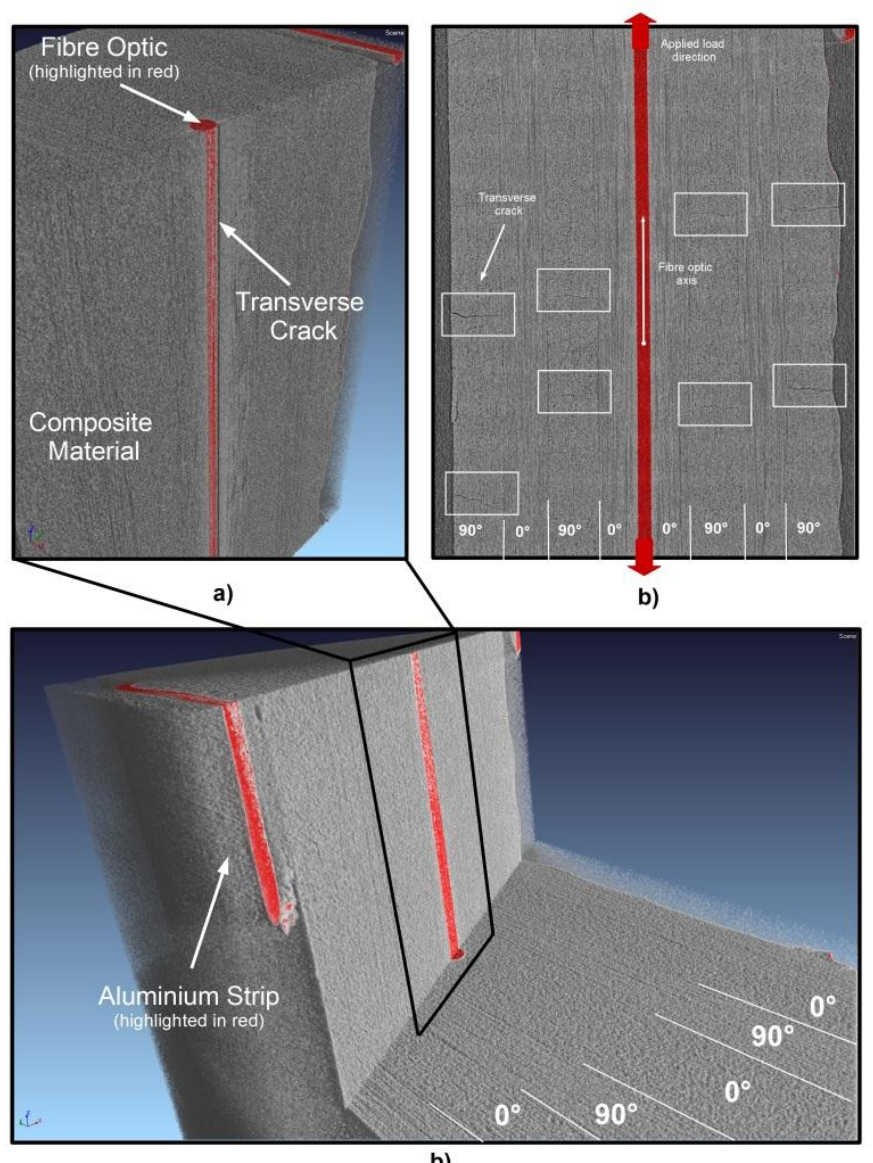

b)

Figure 7. (c) 3D rendering of a CT scan on the fibre optics surroundings for a carbon fibre sample, (a) detail of the crack evolving through the whole fibre coating and (b) cross-section taken along the fibre axis; the matrix cracks are indicated in white boxes.

In order to quantitatively assess the damage evolution over 4,000,000 fatigue cycles, the overall num- ber of cracks lying on a section taken along the fibre optic axis has been tracked for each scan. In addition to this, the stress-strain curve (i.e. sample stiffness) has been determined at each static tensile test. The results for the laminate longitudinal elastic modulus evaluated at different fatigue cycles for a M55J-M18 sample are presented in Table 1. Although not always evident for all the tested samples, here a clear correspondence between stiffness degradation and transverse cracks development could be noticed.

\begin{tabular}{cccc}
\hline cycles nr. & $\sigma_{\max }[\mathrm{Mpa}]$ & $\mathrm{E}_{11}[\mathrm{GPa}]$ & cracks nr. \\
\hline 1,000 & 450 & 144.18 & 3 \\
$1,000,000$ & 450 & 141.35 & 12 \\
$1,000,001$ & 600 & 127.68 & 13 \\
$2,000,000$ & 600 & 121.88 & 20 \\
\hline
\end{tabular}

Table 1. Laminate stiffness degradation and overall number of transverse cracks for a M55J-M18 sample tested over 2,000,000 fatigue cycles.

\section{CONCLUSION}

In this study the potential of the non-destructive technique known as High-Resolution X-ray Computed Tomography or $\mu \mathrm{CT}$ was presented for internal material investigation on CFRP and GFRP composite laminates. The scanned samples had fibre optics embedded, and the $\mu \mathrm{CT}$ proved capable of clearly visualizing the fibre outlines even for GFRP, where the attenuation difference between the materials is small (i.e. reinforcing glass fibre/silica optical fibre have comparable density, therefore similar attenuation coefficients). Moreover, the technique was exploited to assess the quality of in-composite embedded fibre optics. This has revealed in some cases internal damage on the fibre optic surroundings already after manufacturing of the laminates. The samples were then subjected to tension-tension fatigue and cycled to failure. Despite this initial damage, no significant reduction of the fatigue lifetime was encountered for the samples having fibre optics embedded. Even for the "already damaged samples", no further crack growth on the sensor surroundings was noticed after 4,000,000 cycles.

\section{ACKNOWLEDGMENTS}

The research leading to these results has received funding from the Flemish Agency for Innovation by Science and Technology (IWT) - through the program for Strategic Basic Research (SBO) under grant agreement $\mathrm{n}^{\circ} 120024$ (Self Sensing Composites). The author also gratefully acknowledges the significant support of UGCT - the "Centre for X-ray Tomography" of Ghent University. The Special Research Fund of the Ghent University (BOF) is 
acknowledged for the post-doctoral grant of M. N.

Boone.

\section{REFERENCES}

Berthelot J., El Mahi A. \& Le Corre J.F., 2001. Development of transverse cracking in cross-ply laminates during fatigue tests, Composites Science and Technology 61(12): 17111721.

Crupi V., Epasto G. \& Guglielmino E., 2011. Computed Tomography analysis of damage in composites subjected to impact loading, Frattura ed Integrità Strutturale, 17: 32-41.

Dierick M., Van Loo D., Masschaele B., Van den Bulcke J., Van Acker J., Cnudde V., Van Hoorebeke L., 2014. Recent micro-CT scanner developments at UGCT, Nuclear Instruments and Methods in Physics Research B, 324: 35-40.

Hahn H. T., 1979. Fatigue behavior and life prediction of composite laminates, Composite materials: testing and design; Proc. fifth conference ASTM STP 674, 383-417, 20-22 March 1978, New Orleans.

Hufenbach W., Bohm R., Gude M., Berthel M., Hornig A., Ručevskis S. \& Andrich M., 2012. A test device for damage characterization of composites based on in situ computed tomography, Composites Science and Technology 72: 1361-1367.

Ketterer J., 2009. Fatigue crack initiation in cross-ply carbon fiber laminates, Georgia Institute of Technology.

Luyckx G., Voet E., Lammens N. \& Degrieck J., 2011. Strain Measurements of Composite Laminates with Embedded Fibre Bragg Gratings: Criticism and Opportunities for Research, Sensors, 11: 384-408.

Masschaele B.C., Cnudde V., Dierick M., Jacobs P., Van Hoorebeke L. \& Vlassenbroeck J., 2007. UGCT: New xray radiography and tomography facility, Nuclear Instruments \& Methods in Physics Research Section aAccelerators Spectrometers Detectors and Associated Equipment, 580(1): 266-269.

Masschaele B., Dierick M., Cnudde V., Van Loo D., Boone M., Brabant L., Pauwels E., Cnudde V., Van Hoorebeke L., 2013. HECTOR: A 240kV micro-CT setup optimized for research, Journal of Physics: Conference Series 463; Proc. 11th International Conference on X-ray Microscopy, 5-10 August 2012, Shanghai.

Shivakumar K. \& Emmanwori L., 2004. Mechanics of failure of composite laminates with an embedded fiber optic sensor, Journal of Composite Materials, 38(8): 669-680.

Sisodia S., Kazemahvazi S., Zenkert D., Edgren F. , Fatigue Testing Of Composites With In-Situ Full-Field Strain Measurement, Proc. ICCM-18, Jeju Island, Korea, 21-26 August, 2011.

Takeda N., 2002. Characterization of microscopic damage in composite laminates and real-time monitoring by embedded optical fiber sensors, International Journal of Fatigue, 24: 281-289.

Vlassenbroeck J., Dierick M., Masschaele B., Cnudde V., Hoorebeke L. \& Jacobs P., 2007. Software tools for quantification of X-ray micro-tomography, Nuclear Instruments \& Methods in Physics Research Section a-Accelerators Spectrometers Detectors and Associated Equipment, 580(1): 442-445. 